\title{
Implications of Low Serum Albumin as a Prognostic Factor of Long-term Outcomes in Patients With Breast Cancer
}

\author{
TAKAAKI FUJII, SHOKO TOKUDA, YUKO NAKAZAWA, SASAGU KUROZUMI, \\ SAYAKA OBAYASHI, REINA YAJIMA and KEN SHIRABE \\ Division of Breast and Endocrine Surgery, Department of General Surgical Science, \\ Graduate School of Medicine, Gunma University, Gunma, Japan
}

\begin{abstract}
Background/Aim: We examined the relationship between preoperative serum albumin levels and long-term outcomes in patients with breast cancer. Patients and Methods: We retrospectively analyzed the records of 157 patients who underwent breast cancer surgery at a single institution. We divided the patients into those with $<4.0 \mathrm{~g} / \mathrm{dl}$ and those with $\geq 4.0 \mathrm{~g} / \mathrm{dl}$ preoperative serum albumin. Results: The overall median follow-up period was 86.7 months. Among the 157 patients, 19 (12.1\%) had decreased serum albumin levels preoperatively. A significant association with preoperative albumin levels was found only for patient age; however, we were unable to determine an association between preoperative albumin levels and various clinical features. The recurrence-free survival $(p=0.030)$ and the overall survival $(p=0.001)$ were both significantly shorter in patients with low albumin levels. Conclusion: Low serum albumin levels were associated with poor prognosis, but not with poor-prognostic factors. Therefore, low albumin levels may reflect the tumor microenvironment in breast cancer.
\end{abstract}

Cancer frequently effects the nutritional status of cancer patients. An increasing amount of evidence indicates that the patient's preoperative nutritional status is associated with poor survival in various types of cancer (1-3), and several nutritional factors have been investigated for their possible value in predicting a poor outcome (1-12). The serum albumin level is a valuable marker for assessing patients'

This article is freely accessible online.

Correspondence to: Takaaki Fujii, MD, Ph.D., FACS, Department of General Surgical Science, Graduate School of Medicine, Gunma University, 3-39-22 Showa-machi, Maebashi, Gunma 371-8511, Japan. Tel: +81 0272208224, Fax: +81 0272208230, e-mail: ftakaaki@gunma-u.ac.jp

Key Words: Albumin, breast cancer, prognosis, inflammation, immunity. nutritional status in clinical practice (13). The serum albumin level has been re-emphasized by extending its clinical use to determine the severity of disease, disease progression, and prognosis in various types of cancer (1-3, 8-12). We have also reported that albumin is an indicator of the risk for recurrent disease in colorectal cancer (3).

However, in breast cancer patients, we were unable to confirm an association between preoperative albumin and disease recurrence, as the follow-up periods were short (2). We conducted the present study to examine the relationship between preoperative serum albumin and long-term outcomes in patients with breast cancer as part of our research concerning the effects of a malnutrition state or other factors on cancer patient outcomes.

\section{Patients and Methods}

Patients. We retrospectively analyzed the records of 157 consecutive patients treated for breast cancer at Gunma University between March 2007 and December 2013. All patients had already undergone radical breast surgery. Patients with synchronous bilateral breast cancer or clinical signs of infection or other inflammatory conditions preoperatively, including pneumonia or rheumatoid arthritis, were excluded. Patients with incomplete clinical information and those who received neo-adjuvant chemotherapy were also excluded. Written consent was obtained from all patients for the use of their records and imaging in future studies, and this was approved by our Clinical Ethics Committee.

The details extracted from the database were the patient's age, tumor's histological type, size of the invasive primary tumor, axillary lymph node status, presence of lymphatic/vascular invasion, nuclear grade, estrogen receptor (ER) expression status and progesterone receptor $(\mathrm{PgR})$ expression status, human epidermal growth factor receptor 2 (HER2) score of the primary tumor, serum C-reactive protein (CRP) level, presence/absence of the serum tumor marker carcinoembryonic antigen (CEA), and serum albumin levels. The ER and PgR statuses were assessed by the Allred score, with an Allred score of $\geq 3$ indicating ER and PgR positivity (14). The overall median follow-up period was 86.7 months, and none of the patients died of surgical complications.

Blood samples were obtained preoperatively. In an adult, the normal range of serum albumin is defined as 4.0-5.0 g/dl, and levels 
Table I. Patients characteristics and clinicopathological features associated with preoperative serum albumin level.

\begin{tabular}{lccr}
\hline & \multicolumn{2}{c}{ Serum albumin level } & \\
\cline { 2 - 3 } & Low (n=19) & High (n=138) & $p$-Value \\
\hline Age (y.o.) & $67.6 \pm 13.5$ & $57.3 \pm 11.6$ & $<0.001$ \\
Histological type & & & 0.542 \\
$\quad$ Invasive ductal & 18 & 110 & \\
$\quad$ carcinoma & & & \\
$\quad$ Non-invasive ductal & 1 & 9 & \\
$\quad$ carcinoma & & & \\
$\quad$ Others & 1 & 18 & \\
Tumor size (mm) & $18.9 \pm 9.1$ & $22.0 \pm 17.5$ & 0.774 \\
Node metastases & $6(31.6 \%)$ & $46(33.3 \%)$ & 0.552 \\
positive (n) & & & \\
ER positive (n) & $15(78.9 \%)$ & $115(83.3 \%)$ & 0.419 \\
PgR positive (n) & $13(68.4 \%)$ & $106(76.8 \%)$ & 0.294 \\
HER2 positive (n) & $6(31.6 \%)$ & $23(16.7 \%)$ & 0.964 \\
Nuclear grade 3 (n) & $10(52.6 \%)$ & $53(38.4 \%)$ & 0.174 \\
ly positive (n) & $6(31.6 \%)$ & $54(39.1 \%)$ & 0.356 \\
v positive (n) & $1(5.3 \%)$ & $19(13.8 \%)$ & 0.265 \\
CEA & $2.2 \pm 1.2$ & $2.3 \pm 2.0$ & 0.584 \\
\hline
\end{tabular}

Values are expressed as mean \pm SD. N: Number; CEA: carcinoembryonic antigen.

less than $3.5 \mathrm{~g} / \mathrm{dl}$ are described as hypoalbuminemia. There were only two cases $(1.3 \%)$ of hypoalbuminemia in the present patient series; we therefore used $4.0 \mathrm{~g} / \mathrm{dl}$ as the cut-off value of serum albumin. We divided the patients into two groups based on their albumin values: low $(<4.0 \mathrm{~g} / \mathrm{dl})(\mathrm{n}=19)$ and high $(\geq 4.0 \mathrm{~g} / \mathrm{dl})(\mathrm{n}=138)$ groups. The relationships between albumin and clinicopathological features were investigated.

Statistical analyses. The breast cancer cases were divided on the basis of their albumin levels. We conducted a univariate statistical analysis using Fisher's exact test or the $c^{2}$ test with Yates' correction. For the comparisons of pairs of groups, we used Student's $t$-test. The recurrence-free survival (RFS) and overall survival (OS) were calculated using the Kaplan-Meier method. The log-rank test was used to evaluate differences between the survival intervals. Differences were considered significant when $p<0.05$.

\section{Results}

Albumin concentrations and clinicopathological features. We analyzed the records of 157 patients who underwent elective breast surgery. The mean age of the patients was $57.8 \pm 12.7$ years, with an age distribution of 28-85 years. The mean preoperative serum albumin was $4.2 \pm 0.3 \mathrm{~g} / \mathrm{dl}$. Of the 157 cases, 19 (12.1\%) had decreased serum albumin (Table I).

Table I summarizes the characteristics of the patients in the two albumin groups and presents the results of the univariate analysis conducted to determine the relationships between preoperative albumin values and the clinicopathologic variables. A significant association with
A

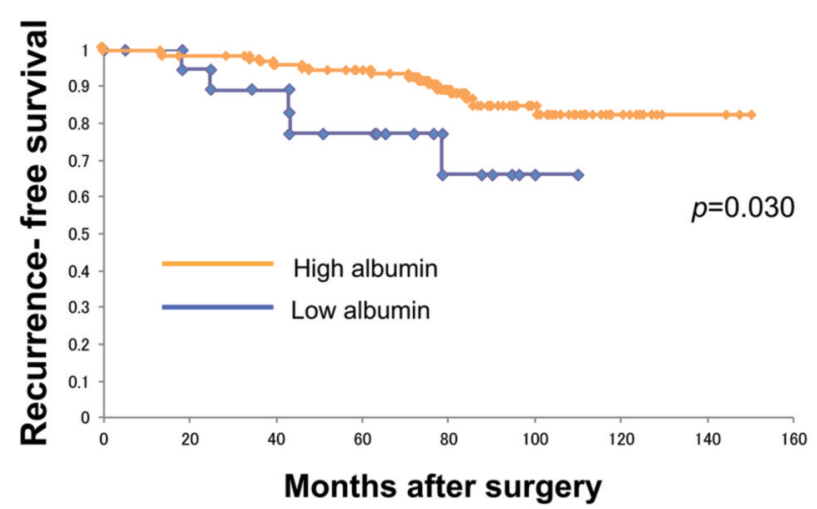

B

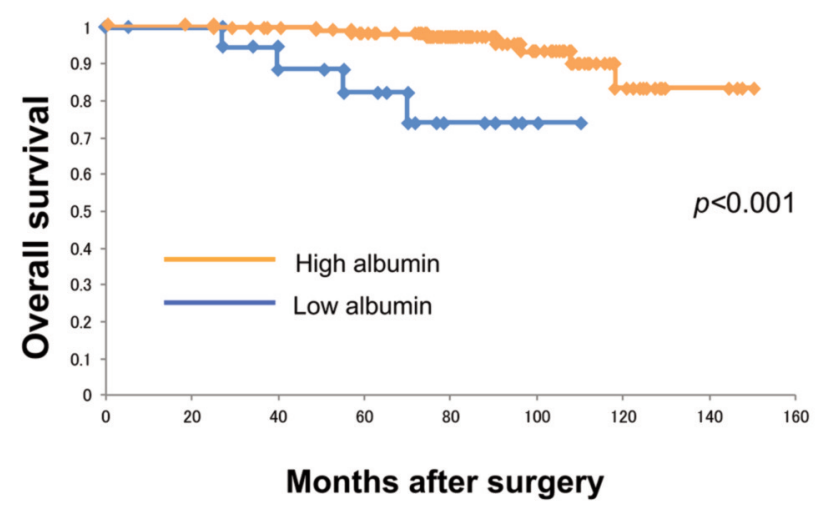

Figure 1. The relationship between low preoperative serum albumin levels and the postoperative recurrence-free survival (RFS) and overall survival $(O S)$ in patients with breast cancer. A: The RFS by KaplanMeier curves was significantly shorter in patients with low serum albumin ( $p=0.030)$. B: The OS was also significantly shorter in patients with low serum albumin $(p<0.001)$.

preoperative albumin levels was found only for patient age, which was significantly higher in the group of patients with low albumin levels (Table I). In short, no association between preoperative albumin and various clinical features was observed.

The RFS period estimated by Kaplan-Meier curves was significantly shorter in the patients with low albumin $(p=0.030)$ (Figure 1A). The OS period was also significantly shorter for the patients with low albumin $(p=0.001)$ (Figure 1B). The overall median follow-up period was 86.7 months (range $=5.1-147.3$ months).

\section{Discussion}

Serum albumin is indispensable for the physiological activities of the human body and is known to be a nutritional indicator. Low serum albumin reflects a state of malnutrition. 
It is of interest that malnutrition has been reported to be associated with poor prognoses in patients with multiple types of cancer (1-12). Malnutrition in patients with cancer is a significant problem due to a variety of mechanisms involving the tumor and the host response to the tumor.

The key observation made in the present study is that low preoperative serum albumin was associated with poor RFS and OS in patients with early breast cancer. Low serum albumin levels have been reported to be a predictor of poor outcomes in patients with various malignant tumors (1-3, 8-12), and we have also observed that albumin was a sensitive indicator of the risk for recurrent disease in colorectal cancer (3). These findings provide evidence that the serum albumin levels are likely to be a predictor of prognosis in patients with breast cancer.

Serum albumin is also an indicator of morbidity and mortality. The interpretation of serum albumin is often difficult because non-nutritional factors can obscure the effects of actual nutrient deprivation. The body's serum albumin levels are influenced by various factors, such as stress, liver failure, aging, hydration state, and disease processes $(1,15)$. The serum albumin levels also decrease due to inflammation. Furthermore, low serum albumin concentration may be due to the production of cytokines such as interleukin (IL)-6, which modulate the production of albumin by hepatocytes $(1,16)$. As part of the systemic inflammatory response to a tumor, proinflammatory cytokines and growth factors that may regulate albumin synthesis are released. Several research groups have focused on the correlation between inflammation and solid malignancies, and have revealed that tumor initiation, progression, and metastasis are all affected by the host systemic inflammatory response as well as the tumor microenvironment $(17,18)$. There is slight or no hypoalbuminemia in the early stages of cancer, but as the disease progresses the albumin levels drop significantly and serve as a good indicator of prognosis $(1,12,12)$.

In the present study, there were no clear relationships between the patients' preoperative albumin levels and various clinical features including poor prognostic factors, such as a large tumor size and the presence of node metastasis. In addition, we have observed only slight or no hypoalbuminemia in patients with breast cancer, including the patients with metastatic disease in our previous study (2). Herein, low preoperative serum albumin was associated with poor RFS and OS in patients with early breast cancer. These results imply that low serum albumin may also reflect other mechanisms associated with tumor progression or inflammation. Low albumin in the serum would weaken cellular and humoral immunity, phagocytic functions, and other defense mechanisms in patients with cancer; low albumin may reflect immunological environments (15).

Disease progression in cancer is dependent on the complex interactions between the tumor and the host immunological response (19). Low albumin provides biological information about a tumor's potential outcome. Breast cancer progression is relatively slow; thus, albumin may be ideal for assessing the tumor microenvironment for not only the nutritional status but also the patient's status over a long period of time. Albumin levels have been reported to be associated with breast cancer risk and cancer mortality (20). Further research is necessary to establish the effect of low serum albumin on immunological features and the tumor microenvironment in breast cancer.

This study has several potential limitations, including its retrospective design and the relatively small number of patients $(n=157)$. However, to the best of our knowledge, this is the first study with long follow-up periods to describe the relationship between a low albumin levels and long-term prognosis in breast cancer. Additional research is needed to explore the usefulness of albumin levels as a predictor of the status of the tumor microenvironment in patients with breast cancer.

In conclusion, in the present series of patients with breast cancer, low serum albumin levels were associated with poor prognosis. Low serum albumin levels were not associated with poor prognostic factors, which may reflect the tumor microenvironment in breast cancer. Additional studies are warranted to evaluate how low albumin levels influence the tumor microenvironment in patients with breast cancer.

\section{Conflicts of Interest}

The Authors declare that they have no competing financial interests related to this study.

\section{Authors' Contributions}

TF analyzed data and wrote the initial draft of the manuscript. TF, ST, YN, SK, SO and RY collected data and were involved in the initial study conception and design. TF and KS interpreted the results and were involved in drafting the work and revising it critically for important intellectual content. TF approved the final version to be published. All Authors have read and approved the final manuscript.

\section{Acknowledgements}

The Authors would like to thank Ms. Saitoh Y, Ms. Takata F and Ms. Kanai H for their secretarial assistance. Supported by Grantsin-Aid from the Japanese Ministry of Education, Culture, Sports, Science and Technology (T.F.). This study is supported by Grantsin-Aid from the Japanese Ministry of Education, Culture, Sports, Science and Technology (T.F.).

\section{References}

1 Gupta D and Lis CG: Pretreatment serum albumin as a predictor of cancer survival: A systematic review of the epidemiological literature. Nutr J 9: 69, 2010. PMID: 21176210. DOI: 10.1186/ 1475-2891-9-69 
2 Fujii T, Yajima R, Takada T, Sutoh T, Morita H, Yamaguchi S, Tsutsumi S and Kuwano H: Serum albumin and prealbumin do not predict recurrence in patients with breast cancer. Anticancer Res 34: 3775-3779, 2014. PMID: 24982401.

3 Fujii T, Sutoh T, Morita H, Katoh T, Yajima R, Tsutsumi S, Asao $\mathrm{T}$ and Kuwano H: Serum albumin is superior to prealbumin for predicting short-term recurrence in patients with operable colorectal cancer. Nutr Cancer 64: 1169-1173, 2012. PMID: 23163845. DOI: $10.1080 / 01635581.2012 .718034$

4 Mohri T, Mohri Y, Shigemori T, Takeuchi K, Itoh Y and Kato T: Impact of prognostic nutritional index on long-term outcomes in patients with breast cancer. World J Surg Oncol 14: 170, 2017. PMID: 27349744. DOI: 10.1186/s12957-016-0920-7

5 Mantzorou M, Koutelidakis A, Theocharis S and Giaginis C: Clinical value of nutritional status in cancer: what is its impact and how it affects disease progression and prognosis? Nutr Cancer 69: 1151-1176, 2017. PMID: 29083236. DOI: 10.1080/ 01635581.2017 .1367947

6 Morgan TM, Tang D, Stratton KL, Barocas DA, Anderson CB, Gregg JR, Chang SS, Cookson MS, Herrell SD, Smith JA Jr and Clark PE: Preoperative nutritional status is an important predictor of survival in patients undergoing surgery for renal cell carcinoma. Eur Urol 59: 923-928, 2011. PMID: 21295907. DOI: 10.1016/j.eururo.2011.01.034

7 Barber MD, Ross JA and Fearon KC: Changes in nutritional, functional, and inflammatory markers in advanced pancreatic cancer. Nutr Cancer 35: 106-110, 1999. PMID: 10693162. DOI: 10.1207/S15327914NC352_2

8 Lai CC, You JF, Yeh CY, Chen JS, Tang R, Wang JY and Chin CC: Low preoperative serum albumin in colon cancer: a risk factor for poor outcome. Int J Colorectal Dis 26: 473-481, 2011. PMID: 21190025. DOI: 10.1007/s00384-010-1113-4

9 Onate-Ocana LF, Aiello-Crocifoglio V, Gallardo-Rincon D, Herrera-Goepfert R, Brom-Valladares R, Carrillo JF, Cervera E and Mohar-Betancourt A: Serum albumin as a significant prognostic factor for patients with gastric carcinoma. Ann Surg Oncol 14: 381-389, 2007. PMID: 17160496. DOI: 10.1245/ s10434-006-9093-x

10 Lis CG, Grutsch JF, Vashi PG and Lammersfeld CA: Is albumin an independent predictor of survival in patients with breast cancer? J Parenter Enteral Nutr 27: 10-15, 2003. PMID: 12549592. DOI: $10.1177 / 014860710302700110$

11 McMillan DC, Watson WS, O'Gorman P, Preston T, Scott HR and McArdle CS: Albumin concentrations are primarily determined by the body cell mass and the systemic inflammatory response in cancer patients with weight loss. Nutr Cancer 39: 210-213, 2001. PMID: 11759282. DOI: 10.1207/S15327914nc392_8
12 Miura K, Hamanaka K, Koizumi T, Kitaguchi Y, Terada Y, Nakamura D, Kumeda H, Agatsuma H, Hyogotani A, Kawakami S, Yoshizawa A, Asaka S and Ito KI: Clinical significance of preoperative serum albumin level for prognosis in surgically resected patients with non-small cell lung cancer: comparative study of normal lung, emphysema, and pulmonary fibrosis. Lung Cancer 111: 88-95, 2017. PMID: 28838406. DOI: 10.1016/j.lungcan.2017.07.003

13 von Meyenfeldt $\mathrm{M}$ : Cancer-associated malnutrition: an introduction. Eur J Oncol Nurs 9: S35-S38, 2005. PMID: 16437756. DOI: 10.1016/j.ejon.2005.09.001

14 Allred DC, Harvey JM, Berardo M and Clark GM: Prognostic and predictive factors in breast cancer by immunohistochemical analysis. Mod Pathol 11: 155-168, 1998. PMID: 9504686.

15 Nakanishi Y, Masuda T, Yamaguchi K, Sakamoto S, Horimasu Y, Mimae T, Nakashima T, Miyamoto S, Tsutani Y, Iwamoto H, Fujitaka K, Miyata Y, Hamada H, Okada M and Hattori N: Albumin-globulin ratio is a predictive biomarker of antitumor effect of anti-PD-1 antibody in patients with non-small cell lung cancer. Int J Clin Oncol 25: 74-81, 2020. PMID: 31531785. DOI: $10.1007 / \mathrm{s} 10147-019-01539-2$

16 Delmore G: Assessment of nutritional status in cancer patients: widely neglected? Support Care Cancer 5: 376-380, 1997. PMID: 9322349. DOI: 10.1007/s005200050095

17 Guo W, Lu X, Liu Q, Zhang T, Li P, Qiao W and Deng M: Prognostic value of neutrophil-to-lymphocyte ratio and plateletto-lymphocyte ratio for breast cancer patients: An updated metaanalysis of 17079 individuals. Cancer Med 8: 4145-4148, 2019. PMID: 31197958. DOI: 10.1002/cam4.2281

18 Fujii T, Yanai K, Tokuda S, Nakazawa Y, Kurozumi S, Obayashi $S$, Yajima R, Hirakata T and Shirabe K: Relationship between FDG uptake and neutrophil/lymphocyte ratio in patients with invasive ductal breast cancer. Anticancer Res 38: 4927-4931, 2018. PMID: 30061271. DOI: 10.21873/anticanres.12809

19 Roxburgh CS and McMillan DC: Role of systemic inflammatory response in predicting survival in patients with primary operable cancer. Future Oncol 6: 149-163, 2010. PMID: 20021215. DOI: $10.2217 /$ fon. 09.136

20 Kühn T, Sookthai D, Graf ME, Schübel R, Freisling H, Johnson T, Katzke V and Kaaks R: Albumin, bilirubin, uric acid and cancer risk: results from a prospective population-based study. Br J Cancer 117: 1572-1579, 2017. PMID: 28898231. DOI: $10.1038 /$ bjc. 2017.313

Received April 9, 2020

Revised April 18, 2020

Accepted April 20, 2020 